\title{
Phoswich scintillator assemblies: Application to the Simultaneous detection of Particle and Gamma radiation - PASPAG
}

\author{
O. Tengblad, M. Garcia Castaño, I. Marroquin \\ Instituto de Estructura de la Materia CSIC, Serrano 113, ES-28006 Madrid, Spain
}

\section{A R T I C L E I N F O}

\section{Keywords:}

Scintillators

Gamma-detection

Particle-detection

Applications

Homeland security

\begin{abstract}
A B S T R A C T
PASPAG is a Joint Research Activity within the Horizon2020-project ENSAR2 [1]. PASPAG aims to improve the detection system for European Large Scale Facilities to make best use of the high investment in delivering radioactive ion beams. Efficient gamma-ray and charged-particle detection are key tools for experimental nuclear physics. PASPAG aims for simultaneous detection of gamma and particle radiation by the use of new scintillator materials combined with the phoswich technique.

In this report we first present the different tasks within the PASPAG-project in order to continue with a more detailed discussion on the R\&D towards societal applications that are performed within the project. Especially we define the needed characteristics of detection systems for homeland security defined by the Thematic group for Radiological and Nuclear threats within the European Reference Network for Critical Infrastructure Protection (ERNCIP) [2].
\end{abstract}

\section{PASPAG}

The PASPAG collaboration exploits novel scintillator materials and explores new techniques and concepts such as phoswich detectors and segmented or hybrid scintillators in order to develop the capability to simultaneously detect high-energy gamma rays, neutrons and charged particles [3]. The emphasis is on a modular approach both in the scintillator crystals and photosensors as well as in the electronics where improved throughput and effective data processing will allow for compact scalable devices.

PASPAG takes this technology out of basic science so that it can be exploited for societal applications within, for example, the areas of nuclear medicine and homeland security. Depending on the applications, features like energy resolution, position sensitivity, high rate capability, and insensitivity to magnetic fields or radiation hardness are of differing importance. PASPAG is organized into different tasks, strongly connected with each other that range from the identification and characterization of new materials to the construction of small-size phoswich prototypes and hybrid detectors, to be used in applications.

\section{Novel Scintillator Materials}

A wide range of promising new scintillators are becoming commercially available, such as $\mathrm{CeBr}_{3}$, CLYC, GAGG, GYGAG:Ce, CLLB, CLLC [4-6]. It has also recently been discovered that co-doping inorganic scintillators might increase the crystal proportionality and significantly improve the energy resolution [7]. It is not clear, however, how these developments in scintillator performance might translate into practical applications for nuclear physics and the new materials need to be characterized in this regard.

The ideal scintillator should provide not only a high light yield but also a high effective atomic number for good stopping power, a short decay time constant for fast response, and a good level of linear response for good energy resolution. In addition, chemical and mechanical robustness are needed to allow the scintillator detector to be used in many different applications and environments. Fig. 1 displays a schematic ordering of the existing materials as a function of the expected energy resolution that can be obtained with each of them. A strand of this project investigates hybrid detector arrays, where we are to combine different scintillator materials and readout sensors.

\section{Hybrid arrays and their applications}

By hybrid arrays, we mean highly-segmented assemblies of different scintillator materials, and also the combined use of photosensors on the same detector package, for example, position sensitivity achieved with SiPMs on one side and a PMT on the other to obtain the best energy or timing resolution. One kind of hybrid detector is the phoswich-detector where two different scintillators are optically coupled. In order to be able to separate the energy deposited in each of the two parts of the phoswich, the two materials are chosen to have different, emission wave-length and timing properties.

Phoswich solutions are attractive for discriminating high-energy charged particles and gamma rays $[8,9]$. We also address societal applications outside fundamental research, such applications span a broad range from medical imaging to homeland security. 


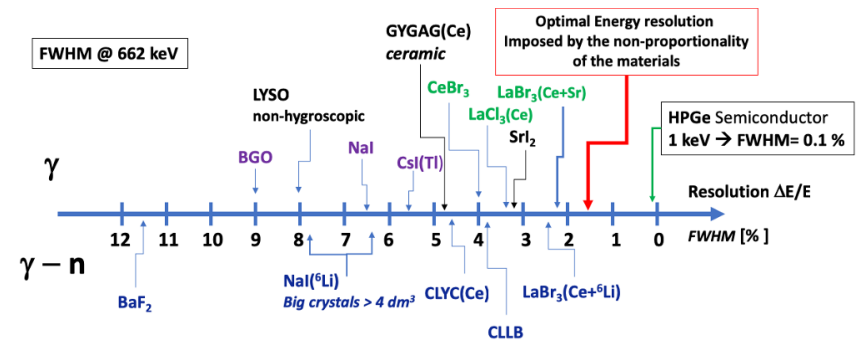

Fig. 1. Existing high resolution scintillator materials ordered according to the energy resolution that can be obtained: FWHM of $\Delta \mathrm{E} / \mathrm{E}$ in $\%$ at $662 \mathrm{keV}$. The figure is compiled from readily available on-line data.

\section{Homeland Security}

The European Internal Security Strategy draws attention to the need to enhance capabilities against CBRNE (chemical, biological, radiological, nuclear, explosives) threats, including developing minimum detection and sampling standards.

Novel detection technologies can impact nuclear security in many ways [10]. Instruments for nuclear security are usually designed for the detection and identification of the radioactive source. However, a key piece of information required for field operations, such as the position of the emitting source is often missing. The capability of an instrument to point to the source can be implemented in various ways. With detectors recording the direction of the incoming radiation or combining several detectors. Today's technique makes it possible to install autonomous detectors that are capable to record and transmit data without human intervention. Combined with reliable data transfer, the final data analysis can be performed in a centralized expert support centre by optimally utilizing data from multiple sensors.

With the new technologies it is possible to develop more efficient detection systems. Especially the digitalization and the Internet of Things (IoT) provide great opportunities for cooperation at the technical level. The IoT communication layer for the detectors and detector networks, enables a continuous low-level on-line automatization of early warning and an efficient high-level expert support in technical and scientific matters. Big Data and related Data Mining applied to the automated on-line monitoring have the potential to analyse observations over longer time periods for early warning and early prevention of prevailing illegal activities.

\section{Improved radiation detection systems for Nuclear Security}

For the application in homeland security features like energy resolution, position sensitivity, high rate capability, and insensitivity to magnetic fields are of importance. Further, for autonomous use, low power-consumption, low weight and compact size are important characteristics. There is also a need for remote-controlled radiation measurements and sampling using unmanned vehicles (robots or drones). There are several measurement and sampling scenarios that are too risky for humans to carry out. Applications envisaged are: reactor and other accidents, dirty bombs before and after explosion, and in general search of sources out of regulatory control. In these cases there is a special need for lightweight detectors and manipulators. A special case is Forensic; in case of malicious activity gathering of proof on a radiological-dirty crime scene has to be handled by drones.

Further, the need for standardization of list-mode data has been recognized for European security standards and a new standard IEC 63047:2018 was recently published [11]. Emphasis should thus be on a modular approach both in the scintillator crystals and photosensors as well as in the electronics with improved throughput and effective data processing that can allow for compact scalable devices.

Digital signal processing together with list-mode data makes it possible to fully take advantage of the sensitivity to both gamma and neutron radiation as well as to take advantage of the imaging possibilities combining different materials in one detector. By adding Time and Geo-stamping of the data in realtime (listmode) one can more easily scan and map big areas e.g. the dose-rate after a nuclear-fallout following a reactor accident, or scan container-storage in a harbour. There is a need for detectors with directional sensitivity and with simultaneous gamma ray and particle identification. The correlation between measurements can be done on short and on long timescale. On the one hand the coincidence measurements between several detectors simultaneously can help in localization. On the other hand transport of radioactive material across countries can be followed passing different border points.

\section{R\&D on New technologies for Nuclear Security}

We are addressing the mentioned ideas by testing different systems to analyze the signals produced in the scintillator when exposed to gamma radiation and tested different types of photo-sensors in order to optimize and reduce the weight of the detector. Especially a Phoswich $\mathrm{LaBr}_{3}(\mathrm{Ce})+\mathrm{LaCl}_{3}(\mathrm{Ce})$ in the form of truncated pyramid (entrance area $15 \times 15 \mathrm{~mm}^{2}$, length $4+6 \mathrm{~cm}$, and exit area $24 \times 24 \mathrm{~mm}^{2}$ ) was mounted with a Hamamatsu S13361-3050AS-08/NG (8x8 pixels of $3 \times 3 \mathrm{~mm}^{2}$ ) SiPM array. The SiPM was powered by $54 \mathrm{~V}$ and taking the combined signal of all 64 pixels via a Mesytec MDS-8 NIM based spectroscopy amplifier a $3.5 \%$ resolution at $1 \mathrm{MeV}$ was easily obtained [3]. One of the possible practical use of the phoswich is for early warning systems, where one would like to know if the radioactivity is deposited on the ground or is still airborne. The system will be equipped with a computer of small size with built-in GEO and TIME-stamping including wireless communication to a base station. The computer found for this purpose is the Raspberry Pi[12]. It is a programmable device, with Linux as the default operating system. It can be equipped with peripherals such as a camera, GPS and incorporates wireless LAN and Bluetooth connectivity, it is small $8 \times 6 \times 2 \mathrm{~cm}$, low operating voltage (5V) and low weight. Using the GPIO (general purpose input output) connects the Pi to physical extensions as ADC (Analog to Digital Converter). The Raspberry Pi comes with pre-installed libraries to access the IO using Python, C or $\mathrm{C}++$. The detected signals are digitized by the ADC and transmitted over a wireless LAN to the base station. It is still necessary to improve the conversion of signals from the detector to spectra to obtain a final product.

\section{Summary}

Novel scintillator materials with $\gamma$-n identification that are insensitive to humidity, magnetic field are emerging, coupled with lightweight low-powered sensors like SiPM are ideal to be used in Nuclear Security.

The PASPAG collaboration has identified societal applications of our R\&D, especially in the case of Nuclear security we are aiming for a lightweight compact detector device that can be carried by an unmanned vehicle (remote-controlled) for measurements in areas of high radiation. It should be equipped with an accurate small sized computer with incorporated geo-location and time-stamping systems for wireless communication to a base station.

There is a growing $R \& D$ activity in this field $[10,13]$ and there are commercial solutions existing that partly fulfil the needs, see for e.g. $[14,15]$.

\section{Acknowledgement}

The ENSAR2 project has received funding from the European Union via the Horizon 2020 research and innovation program under grant agreement No 654002. MGC has received funding from the Community of Madrid via de program "Young Employment Initiative" (YEI) co-financed by the European Social Funds. 


\section{References}

[1] ENSAR2: http://www.ensarfp7.eu.

[2] ERNCIP: https://erncip-project.jrc.ec.europa.eu.

[3] http://www.ensarfp7.eu/activities/joint-research-activities/paspag.

[4] M.Moszynski et al. Nucl. Instr. \& Meth. A805, 25-35, (2016).

[5] T. Yanagida, et al., Optical Materials 35 (2013) 2480?2485.

[6] F. Patrick Doty, et al., Sandia Report SAND2012-9951 (2012).

[7] M.S. Alekhin, et al., P. Journal of Applied Physics 113 (22) (2013) 224904 .

[8] O. Tengblad, et al., Nucl. Instr. and Meth. A704 (2013) 19-26.

[9] E. Nacher, et al., Nucl. Instr. \& Meth. A769 (2015) 105-111.
[10] Applications of Novel Scintillators for Research and Industry, J. Phys.: Conf. Ser. 763 (2016) 011001 .

[11] International Electrotechnical Commission oficial standard: IEC 63047:2018, Nuclear instrumentation - https://webstore.iec.ch/publication/28999.

[12] https://www.raspberrypi.org/products/raspberry-pi-3-model-b/.

[13] Chil R. et al. (2014) Advanced Networked Modular Personal Dosimetry System. In: Roa Romero L. (eds) XIII Mediterranean Conference on Medical and Biological Engineering and Computing 2013. IFMBE Proceedings, vol 41. Springer, Cham.

[14] Icohup https://www.icohup.com/en/.

[15] Mirion https://www.mirion.com/products/radiation-monitoring-services. 